\title{
Editorial: In search of value: taking the reader on a journey to valuable contribution in journal publishing
}

Simon D. Smith BEng, PhD, CEng, FICE

Chair and Honorary Editor, Editorial Advisory Panel; Senior Lecturer, School of Engineering, University of Edinburgh, Edinburgh, UK

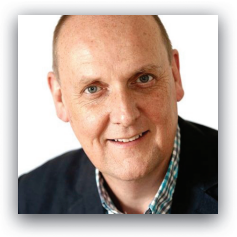

\section{Introduction}

It gives me great pleasure to welcome readers to the first issue of 2020. As Chair of the Editorial Advisory Panel and on behalf of the panel I would like to take this opportunity to expand upon the nature of the journal, its scope, the role of the panel and the editorial process that leads to publication of papers and briefings in this journal. I would like to reflect upon what it is we are looking for in a published paper - and perhaps also the things we are not looking for.

One of the aspects about the Institution of Civil Engineers (ICE) journal Management, Procurement and Law is that we serve two distinct, though in many ways quite connected, readerships - those in academia and practice. This makes the journal a little different to most academic sources. While we wish to publish theoretically grounded reports of robust research investigations, we also wish to publish papers that consider the impact of developments in practice and industry, whether these be case studies of current or recent projects, considerations of current challenges that the UK and international construction industry faces, or reflections on legal and procurement developments that might impact both practice and research. An essential point to make, though, is that regardless of the origin of the paper or briefing article, the robustness of the investigation and the detail of the research and narrative contained must be uppermost in the minds of author, reviewer and editor. We all share a collective responsibility to ensure the papers in this journal are high-quality and impactful contributions to our readership.

There are many qualities of a publishable article in this journal. It is difficult to say which is the most important - a significant message that is poorly prepared will struggle to make the journey through the editorial and review process. On the other hand, a very well written manuscript that reports an item that is either insignificant or has enjoyed detailed, previous coverage is unlikely to have a sufficient degree of need for publication.

However, before I get on to the specifics of a 'good' paper let us briefly consider the editorial process. An author submits a paper to this journal which is then considered by the editorial sub-panel most appropriate. As the journal's title suggests, there are three sub-panels that consider management, procurement and legal issues. The panel chair considers papers within all three areas. Members of your editorial panel therefore act collectively as Associate Editors of the journal and they consider whether the submitted article is of sufficient quality to be sent out to peerreview, taking cognisance of the aspects I'll cover shortly. It is worth noting at this point that in 2019 the journal had a 'deskreject' rate of approximately $30 \%$ - that is, approximately one in every three submitted papers is deemed not suitable for peerreview. Authors of such papers are given brief feedback on why this decision is made and they are free to develop their work and submit again if they wish, though as a new submission. If the consensus is that the article is worthy of peer-review, then the panel will suggest names of possible reviewers and these get invited. The paper is sent out for review and when we receive a minimum of two competent reviews one of the editorial panel members will then make a judgement based upon the reviews received and their own views of the article. While there are a number of different categories of decisions they essentially fall in to three types - if we receive very positive reviews we may accept the paper as it is; if the reviews are very negative we may reject; but the most common is that we ask the authors to make amendments to their manuscript based upon reviewer comments. These may be significant changes and, if so, the paper may need to be reviewed again, usually by the same reviewers; but if the changes required are minor then the editorial panel may be able to make a judgment without review. This continues until an acceptable paper emerges.

In making their judgement, the reviewers and editorial panel will consider many of the following aspects.

\section{Contribution}

An article in this, or indeed any journal, exists not simply as a record of an investigation that an author has conducted but as a means of dissemination, and the receiver of that dissemination needs to find 
value in that paper. This value is its contribution and is one of the first things we look for in submitted manuscripts. This attribute is so important that the ICE actually requires authors to state what their contribution is, but regrettably we find too few manuscripts submitted which do this. The reviewers, editors and, most importantly, readers who may cite your work need to understand what the paper contributes to our field. We need to understand what the impact has already been and what may be seen in the future. We need to understand what difference the paper will make to the practitioners, the policymakers and the academics who are also working in this area. Without a clear understanding of this, without being able to judge what a paper's contribution is, the paper will struggle to be published. In some ways the contribution can be seen as the wrapper in which the paper is contained - it looks forward to the way in which the work will be perceived and used in the future, but it must also look backwards at the reasons why the paper exists in the first place, which brings me to the second attribute of a good paper, that of framing.

\section{Framing}

Why does the paper exist? While ultimately the contribution of the paper is of vital importance, in order to make a judgement on this contribution we need to understand the motivation for the work and why the author has conducted their investigation and reported the way they have. Chan (2020) provides an excellent coverage of this aspect. While he does so in the role of Editor-in-Chief of the journal Construction Management and Economics, his philosophy and guidance are very appropriate to Management, Procurement and Law even though his journal is much more academically oriented and theoretically grounded than ours. Authors, reviewers, editors and readers of all kinds will benefit from his insights. Put simply, the framing makes the case for the paper. The author needs to convince readers that there is a need for the work presented. This might be an engineering problem or practice issue that has a current detriment - something about it that needs attention, whether this be a solution to a problem, a commentary on the application of a piece of policy or perhaps a bringing to attention of or improvement of the communication of vital information. The author needs to identify who the work is for - the beneficiaries - and outline what benefit they are likely to receive - that is, the contribution. In order to make this case the authors need to understand the situation as it exists before the paper is written and make very clear how their paper will address that situation. The reader needs to be taken on the authors' journey from the state of detriment of their chosen topic to that of betterment, and the clearer and more coherent the case the authors make for this the easier it is for the reader (and of course the reviewers and editors) to acknowledge and judge how useful this journey is. Here we can refer to Grant and Pollock (2011) who wrote about what it takes to publish in the Academy of Management Journal - 'first impressions matter'. In making this case the authors need to 'hook' the reader, to convince them to continue reading. In doing so they must effectively provide the context for their work and mobilise the relevant literature to their investigation.

\section{Literature and context}

Academic readers may be familiar with a recent discussion on the international discussion board Cooperative Network of Building Researchers. Part of this discussion focused on why, in built environment research, do we spend so much time considering the literature? In other disciplines, such as mathematics, literature is a relatively much smaller part of the paper, but our field is not like mathematics - we are comprised of many different disciplines considering many different types of work from a global perspective. The author of a new paper must engage with this vast field of built environment literature for two main reasons. They must firstly ensure that the work they are embarking upon is not only relevant to the field but is actually of need. This does not mean that if a subject has been published before it cannot be written about again - far from it, instead the author has the responsibility to ensure they are aware of that previous work and that their own work will contribute to it. There is that word again - the new paper must contribute to the existing discourse and narrative, it must complement it and must ensure it does not simply repeat exactly what has been said before. In their last editorial as editors of Construction Management and Economics, Dainty and Leiringer (2019: p. 693) expanded upon this point - they spoke about the avoidance of publishing on 'well-trodden ground': in other words, where is the contribution in a paper which is already 'replete within the pages of other journals'?

The second reason for authors engaging with the previous literature is to ensure that pertinent and appropriate knowledge is included in their work. When good papers are published, they create the knowledge, the theoretical approaches, the methodological protocols and the conclusions that advance that subject - the author of a new paper must mobilise this accepted wisdom in advancing the subject even further.

\section{Method and methodology}

Let's first get something out of the way - method is not methodology. Unfortunately, too many authors use the word 'methodology' as a kind of glorification of the perhaps more mundane word 'method'. If you are writing about the tools and protocols that were used in a research investigation, then you are reporting your methods. If you are considering instead the most appropriate methods to use, and maybe even doing so with a philosophical consideration of the epistemological and ontological issues your subject matter brings up, then you are considering the methodology. As Chan (2020) puts it, the method is the what you have done, the methodology is the why you have done it. A paper can have both and many do, particularly in a qualitative setting.

But even more quantitative work benefits from ensuring the methods used to investigate the subject are appropriate, and thus the author needs to do two things - they must first choose the most appropriate method, and they must then explain why that method has been chosen. Too often we are provided with little justification in submitted work and the reader, reviewer and editor are therefore 
being expected to have faith in the author and accept that they know what they are doing. As it happens, though, your reviewers also know what they are doing, which is why they have been invited for review in the first place. If they do not see an appropriate choice of methods or suitable justification for the choices, then they will be less inclined to accept. If a reader of a published paper does not see a robust application of appropriate methods, they are less likely to cite that work. It is clearly, therefore, in the interest of authors to ensure this aspect is well covered in their manuscripts.

As long as there are appropriate considerations and justifications, it is not for me as editor to dictate which methods you as an author should use. But I would like to add a note of caution to the use of questionnaire surveys. These are probably the most common tools used in built environment research, possibly because of their convenience and ability to generate a large amount of data from small amounts of effort. In some respects that is fine - collecting the views and approaches of a wide range of people may well be important for furthering the understanding of a subject. But it is the interpretation of these data that is more problematic, and the principal reason is that the researcher has very little control over who is creating these data. The worst approach would be to send out a questionnaire to an uncontrolled sample - a social-media poll or an international mailing list for instance. But even if the sample is pre-selected the researcher still cannot control who and how many will respond, let alone have any kind of view about the accuracy of the returns - and this is before we contend with issues of bias and inappropriate expertise in the respondents. A more subtle issue of questionnaire research is the danger of extrapolating or generalising the results - it is very difficult to argue that the interpretations are applicable to anything other than the sample who have provided the data. A recent submission to this journal reported (in its 'methodology' section) that a questionnaire was distributed to an unknown number of contracting people within a small region of the Middle East. An unknown number of responses was received. And yet the conclusions to this work generalised the findings as being appropriate to a much wider population. To summarise, if you do choose questionnaire surveys then ensure you provide very robust justification for this choice of method.

\section{Subject matter}

I'd like to share a few brief words on the matter of subject choice. On the one hand the scope of this journal is very broad - we publish papers focusing on all aspects of the management, procurement and legal issues of delivering our built environment internationally. I have already said, however, that if a new paper covers a topic that is well covered previously then its chances of publication are reduced. Just because a topic is in scope doesn't mean it will be published.

So, what are the big topics of 2020, which are the ones which our readership is already engaged with and will want to read more? High on this list is likely to be 'Industry 4.0 ' - what can it do for construction and, maybe equally as important, should it be done? On this matter I refer to the upcoming themed issue on 'Ethical and social challenges of Construction 4.0' to be guest edited by Dr Fred Sherratt, but also to McAleenan et al. (2019) from this journal - if we are not careful then we could blindly walk the industry into issues of privacy, surveillance and diminished worker engagement.

I also expect topics that we have seen much of in the last year or so to continue. Social value has seen a rise in publications and is a growing topic that considers that the value of construction goes beyond monetary. In the procurement area, the interpretation and implementation of contract forms generally (such as Charlson (2019)) and the fourth edition of NEC specifically (a good example being in this very issue: Leung and Kwok (2020)) continue to attract interest from our readers and authors, but it could be that more case-study pieces are submitted as projects are seen through to completion. Offsite construction and so-called 'modern methods of construction' are other areas of interest possibly for all of the journal's sub-panels - the management of construction has been dealing with prefabrication and offsite manufacturing for some time but the procurement and contractual issues in this sector have seen little consideration so far.

This editorial is not intended to be a crystal ball but, in brief, other potential topics for 2020 include stakeholder and community engagement; worker well-being and health; public perception of projects and their risks; project complexity; project systems; and finally, and inevitably, we may yet see reports of the impact of Brexit on the UK and European construction industries.

\section{Rounding off - the conclusion}

In my own reviews of papers one of the more common phrases I use is 'provide a conclusion that actually concludes'. It's possibly an unfair comment as the conclusion is one of the most difficult parts of a paper to draft; it is also one of the last and authors may be so relieved finally to get to this point that they spend little time on the closing statements. Another more subtle issue that Chan (2020) points out is that authors may be tempted to benefit from the editorial and review comments to refine their work. This would be a risky strategy - better would be for authors to grasp their opportunity: apart from the abstract and introduction, the conclusion will be the most read part of their paper. It closes the loop and answers the 'so what?' question. Many papers will summarise what they have done and succinctly point out the key results, but these are not conclusions.

Instead we need to see the loop closed on the contribution issue and if authors have done a good job of framing their work and identifying the hoped-for contribution at the front-end of their manuscript then the conclusions at the back-end should be able to point definitively to this - what are the implications and impact of the results? To whom will this paper make a difference? Are the results new or unexpected or different to those previously established and if so, what does this mean now and what might it mean later? Are there any caveats to the work done or limitations within which the work must be interpreted? Is there any further work that must now be 
done to interpret the results fully or perhaps that the work in the paper has revealed is now needed? What should now be done by the reader, by future researchers or policymakers? When my own students struggle with conclusions and the 'so what?' question I suggest they attempt to look forward and try and peer into a future shaped by their work. A poor conclusions section will merely look back at what has gone before, whereas instead we must look forward and try to understand what is to come and how the paper just finished will help with this.

It is my hope that this editorial narrative helps authors - both new and established - as well as reviewers understand what is needed for getting a paper published in this journal.

\section{This issue}

And so, to the papers in this current issue. The first is one which I am particularly pleased to see published - Xu (2020), which considers trust in construction, published an earlier version of this paper at the Association of Researchers in Construction Management (ARCOM) conference in Belfast in September 2018. What we found interesting in that article and led to us inviting a fuller journal paper was that it considers the issue of when trust erodes. No one would argue that trust is not important and yet many will recognise, in many organisational settings, the wider detriment once trust diminishes. Xu identified three mechanisms that help with the repair of trust and the implications for this work are clearly as applicable to the many researchers in this area as well as those in practice who are either engaged with maintaining trust or working out what to do once it is gone.

I would like to make a mention that this is the second paper that has emerged from the ARCOM conference series (the first being Eriksson et al. (2019)). This is a relatively new initiative which sees the best papers from an ARCOM conference that are within the scope of Management, Procurement and Law being invited to develop their paper further for the journal. So far, we have seen two papers published, a further four currently under review and six still in development.

Trust is a concept in our second paper, but considered from a procurement and contractual angle. Leung and Kwok (2020) investigate one of the driving philosophies within the NEC contracts, that of promoting a spirit of mutual trust and co-operation. The authors reflect on just how this might be implemented, recognising that it has become a subject of intense debate from practitioners as well as scholars. Their intention is to review the use of this concept within NEC, how it has been interpreted in a range of cited examples and question if and how the legal implications of this aspect may in due course be ascertained in court, the authors concluding that more clarification and elaboration is needed in future editions of the NEC.

In our final paper in this issue, Karami and Olatunji (2020) consider the concept of value engineering. This is a subject that sees surprisingly little attention, at least not in the pages of Management, Procurement and Law. While it is a close cousin of the broader concept of value management, value engineering focuses more on the function of a product and how the value that derives from this might be improved by changes in engineering approaches. This particular paper focuses on marine projects and considers what might be the best protocols for establishing value engineering within such projects. It does so by way of interrogation of value engineering practitioners in seven different countries.

I hope you enjoy these papers, together with the three book reviews also included, and as ever the journal welcomes feedback and discussions on their content.

\section{REFERENCES}

Chan PW (2020) Revisiting basics: theoretically-grounded interesting research that addresses challenges that matter. Construction Management and Economics 38(1): 1-10.

Charlson J (2019) Briefing: Interpreting contractors' mandated standard of design. Proceedings of the Institution of Civil Engineers Management, Procurement and Law 172(4): 142-145, https://doi. org/10.1680/jmapl.19.00007.

Dainty A and Leiringer R (2019) Maintaining a relevant construction management and economics research community. Construction Management and Economics 37(12): 693-696.

Eriksson PE, Volker L, Kadefors A et al. (2019) Collaborative procurement strategies for infrastructure projects: a multiple-case study. Proceedings of the Institution of Civil Engineers - Management, Procurement and Law 172(5): 197-205, https://doi.org/10.1680/ jmapl.19.00016.

Grant AM and Pollock TG (2011) Publishing in AMJ — part 3: setting the hook. Academy of Management Journal 54(5): 873-879.

Karami H and Olatunji OA (2020) Key value engineering protocols in marine projects. Proceedings of the Institution of Civil Engineers - Management, Procurement and Law 173(1): 21-31, https://doi. org/10.1680/jmapl.19.00010.

Leung RHM and Kwok BCH (2020) How one shall interpret 'spirit of mutual trust and co-operation' in NEC contracts. Proceedings of the Institution of Civil Engineers - Management, Procurement and Law 173(1): 14-20, https://doi.org/10.1680/jmapl.18.00051.

McAleenan P, McAleenan C, Ayers G, Behm M and Beachem Z (2019) The ethics deficit in occupational safety and health monitoring technologies. Proceedings of the Institution of Civil Engineers Management, Procurement and Law 172(3): 93-100, https://doi. org/10.1680/jmapl.18.00027.

Xu J (2020) In the shadow of a negative past: repairing and developing trust in construction projects. Proceedings of the Institution of Civil Engineers - Management, Procurement and Law 173(1): 5-13, https:// doi.org/10.1680/jmapl.19.00030. 